\title{
Chronic Energy Deficiency and its Associated Factors Among Lactating Women in Debre Tabor General Hospital, Northcentral Ethiopia
}

\author{
Melaku Tadege Engidaw*, Alemayehu Digssie Gebremariam, Sofonyas Abebaw Tiruneh, \\ Desalegn Tesfa Asnakew, Bedilu Abebe Abate
}

Public Health Department, College of Health Sciences, Debre Tabor University, Debre Tabor, Ethiopia

Email address:

meltad24@gmail.com (M. T. Engidaw),metsagu@yahoo.com (A. D. Gebremariam), zephab2@gmail.com (S. A. Tiruneh), desalegntesfa50@gmail.com (D. T. Asnakew), bediluab@gmail.com (B. A. Abate)

${ }^{*}$ Corresponding author

\section{To cite this article:}

Melaku Tadege Engidaw, Alemayehu Digssie Gebremariam, Sofonyas Abebaw Tiruneh, Desalegn Tesfa Asnakew, Bedilu Abebe Abate. Chronic Energy Deficiency and its Associated Factors Among Lactating Women in Debre Tabor General Hospital, Northcentral Ethiopia. Journal of Family Medicine and Health Care. Vol. 5, No. 1, 2019, pp. 1-7. doi: 10.11648/j.jfmhc.20190501.11

Received: November 29, 2018; Accepted: January 11, 2019; Published: February 28, 2019

\begin{abstract}
Introduction: Maternal nutrition during pregnancy and/or lactation is a fundamental determinant of maternal and child health. So, it can halt the long-term and irreversible consequences of malnutrition. The burden of undernutrition among women and children became a significant public health problem in low and middle-income countries including Ethiopia. However, lactating mothers in these countries are one of the nutritionally vulnerable groups. There are limited studies conducted to assess the nutritional status of lactating women. So, the aim of this study was to assess the chronic energy deficiency and its associated factors among lactating mothers in Debre Tabor General Hospital, Northcentral Ethiopia. Methodology: An institutional based cross-sectional study was conducted to assess the nutritional status of 266 lactating mothers were selected by systematic random sampling from October 2017 to January 2018 . The data were collected by face to face interview by using a pre-tested and structured questionnaire. The physical measurements were taken at the end of the interview. The bivariate and multivariable logistic regression were used to identify associated factors. The variables with a pvalue $<0.2$ were used to select candidate variables for multivariable logistic regression. A p-value of $\leq 0.05$ was used to declare statistical significance. Result: A total of 251 lactating mothers were included in the study which makes the response rate of $94.36 \%$. The prevalence of chronic energy deficiency among lactating women was $17.9 \%$ (95\% CI $(13.5,23.1)$ ). Women's who had daily laborer husband (AOR: 5.02, 95\%CI $(1.36,18.50)$ ), had not Post Natal Care (PNC) follow up (AOR: 6.39 95\% CI $(1.69,24.13))$, and ate 2 times per day (AOR: $4.87,95 \%$ CI $(1.44,16.51))$ were the contributing factors for the development of chronic energy deficiency. Conclusion: In this study, CED is a medium public health problem. The husband's occupation, postnatal care follow up, and maternal feeding frequency were the contributing factors. Health professionals shall provide health education for pregnant and lactating women's behavior regarding nutrition and health to improve maternal nutrition.
\end{abstract}

Keywords: Chronic Energy Deficiency, Lactating Mothers, Prevalence, Ethiopia

\section{Introduction}

Maternal malnutrition is the problem of many developing countries which lead to acute and chronic diseases, poor healthy development, and loss of productivity at individual and community level at large. Maternal undernutrition includes both macro and micronutrient deficiencies [1]. In Ethiopia, undernutrition is a significant public health problem among lactating and pregnant women till nowadays $[2,3]$.

To satisfy the nutritional requirement of lactating women, appropriate nourishment during pregnancy and lactation is very important for the adequate nutrient reserve to be used when it is inadequate. So, counseling on adequate nutrition during pregnancy is very important to prevent nutrients depletions during lactation [4]. Lactating mothers from low and middle-income countries are considered as a nutritionally 
vulnerable group due to frequent pregnancy, inappropriate care, nurturing the family and workload which intern leads to maternal morbidity and mortality [5].

The development of chronic energy deficiency (CED) among lactating mother is common due to the higher requirement of nutrients [6]. The maternal nutrients depletion occurred due to inadequate intake of nutrients that intern leads to poor nutritional status, poor breast milk quality, and drop child growth and development [7,8].

Dietary adequacy before, during, and after pregnancy will help to prevent CED throughout the life cycle [9, 10]. An acceptable dietary diversity score among lactating mother is important to tackle malnutrition [11].

The prevalence of CED among lactating mothers in different parts of Ethiopia ranges from $17.5 \%$ to $25.6 \%$ [12$16]$.

In Ethiopia, studies suggest that current age of the mother, age at first pregnancy, frequency of ANC visit, delivery area, absence of nutritional information and education in the community, Vitamin A supplementation during postpartum period, consumption of iodized salt and extra meal during lactation, length of breastfeeding, meal frequency, maternal and father educational and occupational status and family size were the major determinant factors for the development of CED among lactating mothers [13-17].

Even if the causes of malnutrition are well-established facts, the magnitude and associated factors differ from area to area. Still Studying the magnitude and associated factors of CED among Lactating mothers is very important to prioritize, design and initiates intervention programs based on the relevant information. So, conducting this study was very important to determine the magnitude and its associated factors of CED among lactating mothers.

\section{Methods and Materials}

\subsection{Study Area}

The study was conducted in Debre Tabor General Hospital, Debre Tabor Town. Debre Tabor is the capital Town of South Gondar Zone which is located 666Kms Northcentral of Addis Ababa, the capital city of Ethiopia. The hospital is providing services for around 2,047,206 peoples $($ male $=1,038,913$ and female $=1,008,293$ ) according to 2007 census. Debre Tabor General Hospital offers health services which include inpatient, outpatient, neonatal intensive care, TB/ leprosy, antenatal care, delivery, postnatal care, and family planning services to the urban as well as the rural communities of South Gondar Zone. The Hospital gives services for 139,787 people and more than one third were adult women in 2016/17 according to the hospital annual report. Currently, this is the only General Hospital in the zone to deliver the basic services for the clients form 15 administrative districts.

\subsection{Study Design and Period}

An institution-based cross-sectional study was conducted from October 2017 to January 2018.

\subsection{Study Population}

All lactating women were included in the study but those who were critically ill during data collection time, pregnant, and with a difficulty to take physical measurements were excluded.

\subsection{Sample Size Determination and Sampling Procedure}

The sample size was determined by using single population proportion formula with the assumption of $19.5 \%$ proportion of $\mathrm{CED} /$ underweight among lactating mother from the most recent study in Adama, Eastern Ethiopia [13], $95 \%$ of confidence level, and $5 \%$ of marginal error. Then, the final sample size became 266 after adding $10 \%$ of the nonresponse rate.

The study participants were selected by using a systematic random sampling technique. First, we estimated the number of lactating women at the site of post-natal care, immunization, family planning, growth monitoring, and pediatrics clinic service users for 4 consecutive months. The expected number of lactating mothers who came for these services was 2011. Then, we calculated the $\mathrm{K}^{\text {th }}$ interval and that was 8 . Then the information was collected at the $8^{\text {th }}$ interval in each health service/care units.

\subsection{Operational Definition}

Chronic Energy Deficiency of a lactating mother: currently breastfeeding mothers for her infant or child had a BMI of $<18.5 \mathrm{~kg} / \mathrm{m}^{2}$ [17].

Minimum Women Dietary Diversity Score (MWDDS): when a lactating mother consumed more than or equal to 5 food group among 10 food groups within 24hours [17, 18].

\subsection{Data Collection Methods}

The interview administered structured questionnaire was used to assess CED and its associated factors. The questionnaire was first prepared in the English and then translated into Amharic, the local language of the study area by linguistic professionals and it was translated back to English by another linguistic professional to check its consistency. Two-days training with pretest for 5 diploma midwives was given on the objective, the relevance of the study, confidentiality of information, respondent's right, informed consent and techniques of the face to face interview and physical measurements of height and weight. The supervisor was following the data collection process throughout the data collection period along with the principal investigator. The measurement of the weight of the lactating mothers was done by using calibrated portable electronic digital scale (Seca, Germany model) and recorded to the nearest $0.1 \mathrm{~kg}$. The height was measured by using a portable height-measuring board with a sliding head bar and recorded the nearest $0.1 \mathrm{~cm}$. These measures of height and weight were done three times without shoes and with a possible light closing. The measure of height and weight was recorded on 
the questionnaire and was later used to calculate the body mass index (BMI).

\subsection{Data Quality Assurance}

The pre-test was done outside of the study area (Nefas Mewucha Primary Hospital) on 20 samples before the actual data collection period and necessary corrections were made on the questionnaire. The weight scale has been calibrated by using $1 \mathrm{~kg}$ standard weight, height measurements were checked with other meter taps. During the data collection time, communication between the data collectors, supervisors and the principal investigator was held on a daily basis to update data collection progress and address problems faced. A definition of concepts and terms had been done clearly with the Amharic language to avoid ambiguity. The supervisors and data collectors were recruited outside the hospital to avoid information bias due to familiarization. The collected data were checked for completeness and consistency by the supervisors and the principal investigator during and after data collection.

\subsection{Study Variables}

The dependent variable was chronic energy deficiency among lactating mothers and the independent variables were socio-demographic factors (address, age, educational status, Marital status, ethnicity, religion, husband educational status, monthly family income), obstetric related variables (age at first pregnancy (in years), number of pregnancy, age at first pregnancy, number of live birth), current admission to therapeutic feeding unit, dietary diversity score, the frequency of maternal feeding, health-related variables (existence of chronic illness and diarrhea) variables, and physical measurements.

\subsection{Data Analysis}

The data were edited, coded and entered to Epidata version 3.1 and exported to SPSS version 21 for cleaning and analysis. The data were analyzed for descriptive, bivariate, and multivariable analysis. The principal component analysis was done to build a wealth index. During binary logistic regression, a variable with a p-value $<0.2$ used to select candidate variables for multivariable logistic regression. In this study, a $p$-value of $\leq 5 \%$ was considered as statistically significant after fitting into multivariable logistic regression. For calculated odd ratios, $95 \%$ of confidence intervals had been used to saw the degree of association. The HosmerLemeshow goodness of fit test was performed on the logistic model for CED of lactating mothers $(\mathrm{P}$-value $=0.652 \%)$.

\subsection{Ethical Consideration}

Ethical clearance and permission were obtained from the Institutional Review Committee of Debre Tabor University and then the written permission was obtained from Debre Tabor General Hospital administrative office. Informed consent was obtained from the study participants. The purpose of the study was explained to them. Lastly; before starting the interview and taking a measurement, we informed each participant that they had the right to withdraw from the study at any time. They were assured Confidentiality was maintained at all levels of the study and was assured about the presentation of the final result at scientific community workshops and publication in a reputable journal without a personal identifier. Mothers with chronic energy deficiency were referred to the respective unit of the hospital by using internal referral slip of the Hospital. Finally, all mothers with chronic energy deficiency and overweight got nutritional education and counseling during data collection time.

\section{Result}

\subsection{Socio-Demographic Characteristics}

A total of 251 lactating mothers were included in the study with the response rate of $94.36 \%$. The mean age \pm SD of the respondent was $29.69 \pm 6.10$ years. From 251, 233 (92.80\%) were Amhara by ethnicity and the rest of them were Oromo (18 (7.20\%)). Majority of the study participants $241(96.0 \%)$ were married and the rest $10(4 \%)$ were divorced. In terms of residency, near to three fourth $(185(73.7 \%))$ were from the urban area. Near to one-third of the respondents were found between the age of 25 and $29 \mathrm{yrs}$ as shown in the table below (Table 1).

Table 1. Socio-demographic and economic characteristics of lactating mothers in Debre Tabor General Hospital January 2018 ( $n=251$ ).

\begin{tabular}{|c|c|c|c|}
\hline Variable & Categories & Frequency & $\%$ \\
\hline \multirow{5}{*}{ Current Age } & $20-24$ & 51 & 20.3 \\
\hline & $25-29$ & 86 & 34.3 \\
\hline & $30-34$ & 53 & 21.1 \\
\hline & $35-39$ & 40 & 15.9 \\
\hline & $40-44$ & 21 & 8.4 \\
\hline \multirow[b]{2}{*}{ Religion } & Orthodox & 214 & 85.3 \\
\hline & Muslim & 30 & 12.0 \\
\hline \multirow{5}{*}{ Respondent's educational status } & unable to read and write & 46 & 18.3 \\
\hline & able to read and write & 46 & 18.3 \\
\hline & primary education & 26 & 10.4 \\
\hline & secondary education & 47 & 18.7 \\
\hline & tertiary education and above & 86 & 34.3 \\
\hline \multirow{2}{*}{ Husband's educational status } & unable to read and write & 25 & 10.0 \\
\hline & able to read and write & 45 & 17.9 \\
\hline
\end{tabular}




\begin{tabular}{|c|c|c|c|}
\hline Variable & Categories & Frequency & $\%$ \\
\hline \multirow{6}{*}{ Respondent's occupation } & primary education & 23 & 9.2 \\
\hline & secondary education & 46 & 18.3 \\
\hline & tertiary education and above & 112 & 44.6 \\
\hline & Merchant & 59 & 23.5 \\
\hline & Government employer & 81 & 32.3 \\
\hline & Housewife & 111 & 44.2 \\
\hline \multirow{4}{*}{ Husband's occupation } & Merchant & 72 & 28.7 \\
\hline & Government employer & 111 & 44.2 \\
\hline & Daily labor & 22 & 8.8 \\
\hline & Farmer & 46 & 18.3 \\
\hline \multirow{5}{*}{ Wealth index } & Very poor & 32 & 12.7 \\
\hline & Poor & 43 & 17.1 \\
\hline & medium & 87 & 34.7 \\
\hline & Rich & 52 & 20.7 \\
\hline & Wealthiest & 37 & 14.7 \\
\hline
\end{tabular}

\subsection{Obstetrics Related Characteristics}

The mean age $\pm \mathrm{SD}$ of lactating mothers at first pregnancy was $21.90 \pm 4.23$ years and the minimum age was 15 years. The mean age of the index child and number of average pregnancy with standard deviation were $11.02 \pm 8.59$ months and $2.30 \pm$ 1.43 respectively. More than fifty percent of respondents were multi-para and more than $90 \%$ were attending antenatal care for the index child as shown in (Table 2).

Table 2. Obstetrics related characteristics of lactating mothers in Debre Tabor General Hospital, January 2018 ( $n=251)$.

\begin{tabular}{|c|c|c|c|}
\hline Variable & Categories & Frequency & $\%$ \\
\hline \multirow{3}{*}{ Age at first pregnancy } & $15-19$ & 84 & 33.5 \\
\hline & $20-24$ & 94 & 37.5 \\
\hline & $25-29$ & 73 & 29.1 \\
\hline \multirow{3}{*}{ Gravidity } & Primi gravida & 91 & 36.3 \\
\hline & Multigravida & 147 & 58.6 \\
\hline & Grand gravida & 13 & 5.2 \\
\hline Number of live birth & $>4$ & 13 & 5.2 \\
\hline \multirow{2}{*}{ Current FP utilization } & Yes & 160 & 63.7 \\
\hline & No & 91 & 36.3 \\
\hline \multirow{2}{*}{ Attending ANC for current child } & Yes & 236 & 94.0 \\
\hline & No & 15 & 6.0 \\
\hline \multirow{2}{*}{ Place of delivery } & Health center & 86 & 34.3 \\
\hline & Hospital & 140 & 55.8 \\
\hline \multirow{2}{*}{ Attending PNC for current child } & Yes & 224 & 89.2 \\
\hline & No & 27 & 10.8 \\
\hline \multirow{3}{*}{ Age of current breastfeeding child in months } & $<6$ & 66 & 26.3 \\
\hline & $6-11$ & 74 & 29.5 \\
\hline & $12-23$ & 111 & 44.2 \\
\hline
\end{tabular}

\subsection{Health-Related Factors}

All most all the respondents had no diarrhea 236 (96.3\%) in the last two weeks and none of the respondents reported any known chronic or acute diseases. None of them were admitted for targeted supplementary feeding or food by prescription programs and have no edema.

\subsection{Dietary Related Characteristics}

The average dietary diversity score (DDS) of lactating mother's \pm SD was $4.96 \pm 0.75$. By using 24-hour dietary recall and 10 food groups, all most all of the lactating mothers had good dietary diversity 246 (98\%). In terms of meal frequency, lactating mothers who ate more than three times were 105 (41.8\%), three times were $106(42.2 \%)$, and two times were $40(15.9 \%)$.

\subsection{The Magnitude of Chronic Energy Deficiency}

The mean \pm SD of weight and height of respondent was $51.30 \pm 6.43 \mathrm{Kg}$ and $1.57 \pm 0.05 \mathrm{~m}$ respectively. The mean \pm SD of BMI was $20.73 \pm 2.35 \mathrm{Kg} / \mathrm{m}^{2}$. The minimum BMI was $16.0 \mathrm{Kg} / \mathrm{m}^{2}$ and the maximum was $32.7 \mathrm{~kg} / \mathrm{m}^{2}$. The overall chronic energy deficiency/underweight was 45 (17.9\%, 95\% CI $(13.5,23.1))$. The magnitude of overweight and normal weight were $15(6.0 \%)$ and 191 (76.1\%) respectively. 


\subsection{Associated Factors of Chronic Energy Deficiency}

In bivariate logistic regression; residency, current age, respondent educational status, husband occupation, current family planning usage status, age at 1st pregnancy, gravidity, ANC follow-up during the pregnancy of index child, PNC follow-up during the pregnancy of index child, current frequency of maternal feeding, number of live-birth and wealth index were significant variables. In multivariable logistic regression analysis; having PNC follow up during index child, maternal current feeding frequency, and husband occupation were the associated factors for the development of chronic energy deficiency among lactating women as shown in the table below (Table 3). Duration of breastfeeding or the age of the child was not significantly associated with CED in this study even if it leads to a higher amount of caloric intake.

Women's who had daily laborer husband were 5 times (AOR: $5.02,95 \%$ CI $(1.36,18.50)$ ) more likely to develop CED as compared to merchant husband. Lactating mothers who had no PNC follow up were 6 times (AOR: 6.39, 95\% CI $(1.69,24.13))$ more likely to acquire CED. Lactating women who ate only 2 times per day were 5 times (AOR: $4.87,95 \%$ CI $(1.44,16.51))$ more likely to had CED as compared to those who ate more than three times per day.

Table 3. Factors associated with chronic energy deficiency among lactating mothers in Debre Tabor General Hospital, January 2018 ( $n=251$ ).

\begin{tabular}{|c|c|c|c|c|c|}
\hline \multirow{2}{*}{ Variable } & & \multicolumn{2}{|c|}{ CED } & \multirow{2}{*}{ COR $(95 \%$ CI $)$} & \multirow{2}{*}{ AOR (95\% CI) } \\
\hline & & Yes & No & & \\
\hline \multirow{2}{*}{ Residency } & Urban & 26 & 159 & 1 & 1 \\
\hline & Rural & 19 & 47 & $2.47(1.26,4.85)$ & $0.19(0.03,1.25)$ \\
\hline \multirow{5}{*}{ Current age } & $20-24$ & 5 & 46 & $0.27(0.07,1.01)$ & $2.32(0.21,25.55)$ \\
\hline & $25-29$ & 13 & 73 & $0.44(0.15,1.35)$ & $3.73(0.54,25.84)$ \\
\hline & $30-34$ & 8 & 45 & $0.44(0.13,1.48)$ & $2.23(0.33,15.19)$ \\
\hline & $35-39$ & 13 & 27 & $1.20(0.38,3.82)$ & $1.91(0.31,8.98)$ \\
\hline & $40-44$ & 6 & 15 & 1 & 1 \\
\hline \multirow{5}{*}{ Respondent education } & Unable to read \& write & 20 & 26 & $15.76(4.94,50.33)$ & $3.02(0.57,16.00)$ \\
\hline & Able to read and write & 11 & 35 & $6.44(1.92,21.62)$ & $1.63(0.34,7.82)$ \\
\hline & Primary education & 5 & 21 & $4.88(1.20,19.78)$ & $1.48(0.27,8.28)$ \\
\hline & Secondary education & 5 & 42 & $2.44(0.62,9.56)$ & $1.04(0.19,5.79)$ \\
\hline & Tertiary and above & 4 & 82 & 1 & 1 \\
\hline \multirow{4}{*}{ Husband occupation } & Merchant & 12 & 60 & 1 & 1 \\
\hline & Government employee & 5 & 106 & $0.24(0.08,0.70)$ & $0.28(0.07,1.12)$ \\
\hline & Daily labor & 10 & 12 & $4.17(1.47,11.82)$ & $* 5.02(1.36,18.50)$ \\
\hline & Farmer & 18 & 28 & $3.21(1.36,7.57)$ & $4.12(0.56,30.39)$ \\
\hline \multirow{2}{*}{ Current F/P status } & Yes & 24 & 136 & 1 & 1 \\
\hline & No & 21 & 70 & $1.70(0.88,3.26)$ & $1.28(0.52,3.17)$ \\
\hline \multirow{3}{*}{ Age at $1^{\text {st }}$ pregnancy } & $15-19$ & 23 & 61 & $3.06(1.27,7.36)$ & $0.86(0.20,3.74)$ \\
\hline & $20-24$ & 14 & 80 & $1.42(0.56,3.59)$ & $1.84(0.50,6.68)$ \\
\hline & $25-29$ & 8 & 65 & 1 & 1 \\
\hline \multirow{3}{*}{ Gravidity } & Primi & 8 & 83 & $0.08(0.02,0.31)$ & $0.07(0.01,1.20)$ \\
\hline & Multi & 30 & 117 & $0.22(0.07,0.70)$ & $0.14(0.01,1.50)$ \\
\hline & Grand & 7 & 6 & 1 & 1 \\
\hline \multirow{2}{*}{ ANC follow-up } & Yes & 39 & 197 & 1 & 1 \\
\hline & No & 6 & 9 & $3.37(1.13,10.00)$ & $0.77(0.16,3.66)$ \\
\hline \multirow{2}{*}{ PNC follow-up } & Yes & 31 & 193 & 1 & 1 \\
\hline & No & 14 & 13 & $6.70(2.88,15.60)$ & *6.39 (1.69, 24.13) \\
\hline \multirow{3}{*}{ The frequency of maternal feeding } & Three times a day & 18 & 80 & $2.30(0.95,5.60)$ & $1.78(0.55,5.70)$ \\
\hline & Two times a day & 19 & 44 & $4.43(1.79,10.92)$ & $* 4.87(1.44,16.51)$ \\
\hline & More than three & 8 & 82 & 1 & 1 \\
\hline \multirow{2}{*}{ Number of live-birth } & $\leq 4$ & 40 & 198 & 1 & 1 \\
\hline & $>4$ & 5 & 8 & $3.09(0.96,9.94)$ & $1.15(0.11,11.86)$ \\
\hline \multirow{5}{*}{ Wealth Index } & Very poor & 3 & 29 & 1 & 1 \\
\hline & Poor & 3 & 40 & $0.72(0.14,3.85)$ & $0.40(0.04,3.46)$ \\
\hline & medium & 13 & 74 & $1.70(0.45,6.40)$ & $1.07(0.18,6.29)$ \\
\hline & Rich & 12 & 40 & $2.90(0.75,11.21)$ & $1.74(0.29,10.51)$ \\
\hline & Wealthiest & 14 & 23 & $5.88(1.51,22.97)$ & $4.34(0.62,30.57)$ \\
\hline
\end{tabular}

$*$ Significant during multivariable analysis $(\mathrm{p}<=0.05), 1=$ reference variable

\section{Discussion}

Different kinds of literature show that undernutrition among reproductive age group women decreased worldwide but it is a major public health problem in Africa and Asai including Ethiopia [19]. The household economic status an important determinant factor to have access to food which is unlikely among women [20,21] which may worsen the situation of undernutrition.

In this study, the prevalence of CED was $17.9 \%, 95 \% \mathrm{CI}$ $(13.5,23.1)$ similar with the study conducted at Wombera woreda and Adama [12, 13] and lower than a study conducted in Dedo and Seqa Chekorsa Districts, Jimma Zone, Ethiopia (40.6\%) [22] and Vietnam [23]. The probable 
reason may be the different socioeconomic status and the dietary habit of the respondents.

lactating mothers who had daily laborer husband were 5 times (AOR: $5.02,95 \%$ CI $(1.36,18.50))$ more likely to develop CED as compared to those who had government employee husband which is similar with a study done in the lowland area of Alamata Ethiopia [16] and India [24, 25]. The reason could be mothers who had daily laborer husband would have low economic status and tasks which may demand extra calories $[15,26]$.

Lactating Mothers who had no PNC follow up were 6 times (AOR: 6.39, 95\% CI $(1.69,24.13))$ more likely to had CED which is similar with the study conducted at Samre Woreda, Ethiopia [14]. The possible justification may be having PNC follow up will increase the access for nutritionrelated knowledge and in turn, it may affect the choice and amount of dietary intake and this [27].

Lactating women who ate at only 2 times per day were 5 times (AOR: 4.87, 95\% CI $(1.44,16.51)$ ) more likely to experience $\mathrm{CED}$ as compared to those who ate more than three times per day which is similar with a study done in the Alamata and Samre Woreda, Ethiopia $[14,16]$. The probable reason might be taking extra meal will increase the amount of energy that can be demanded by lactation [28].

In this study sociodemographic variables like age, wealth status, respondent's occupation, and education were not significant. But in the other studies, these variables were significant and associated with the development of undernutrition among women [29]. Also, other factors related to obstetrics and diet were not significant but these variables were significant in other studies [30]. This variation might be due to the multifactorial cause of undernutrition in developing and developing countries [1, 19, 31].

\section{Conclusion}

The prevalence of chronic energy deficiency among lactating women was a medium public health problem. The factors associated with CED were the husband's occupation, postnatal care follow up, and the frequency of maternal feeding. Health professionals shall provide health education for pregnant and lactating women's behavior regarding nutrition and health care follow up to improve maternal nutrition.

\section{Consent for Publication}

Not applicable to this section.

\section{Availability of Data and Materials}

The dataset is available from the corresponding author. The exported data into SPSS is available from the corresponding author so that we can provide the data at any time during the request.

\section{Competing Interests}

We declare that there is no any competing interest with anyone else.

\section{Funding}

No fund is accepted for this research from any organization.

\author{
Abbreviation \\ ANC: Antenatal Care \\ AOR: Adjusted Odds Ratio \\ BMI: Body Mass Index \\ CI: Confidence Interval \\ DDS: Dietary Diversity Score \\ COR: Crude Odds Ratio \\ DDS: Dietary diversity Score \\ EDHS: Ethiopia Demographic and Health Survey \\ MWDDS: Minimum Women Dietary Diversity Score \\ Kg: Kilogram \\ M: meter \\ PNC: Post Natal Care \\ SD: Standard Deviation \\ SPSS: Statistical Package for Social Science \\ WHO: World Health Organization
}

\section{Authors' Contribution}

All stated authors MTE, ADG, SAT, DTA and BAA were involved in this study from the inception to design, acquisition, analysis, and interpretation of data and drafting of the manuscript.

All the authors read and approved the final manuscript.

\section{Acknowledgements}

We deeply acknowledge all the study participants, all the staffs of the Hospital at all level, supervisors and all data collectors for their cooperation and commitment throughout this study.

\section{References}

[1] Black, R. E., et al., Maternal and child undernutrition: global and regional exposures and health consequences. The lancet, 2008. 371 (9608): p. 243-260.

[2] Amare, B., Nutritional status and dietary intake of urban residents in Gondar, Northwest Ethiopia. BMC Public Health, 2012. 12.

[3] CSA, Ethiopia Demographic and Health Survey 2011 2012: p. 173-187.

[4] Nutrition, D. o. H. D., Guidelines on Maternal Nutrition. South Africa: A manual for Health Care Personnel. 2008.

[5] Asha, K. and S. Salil, Nutrient Intake of Lactating Mothers from Rural areas and urban areas. Indian J Soc Res, 1998. 39 (2). 
[6] Allen, L. H., B vitamins in breast milk: relative importance of maternal status and intake, and effects on infant status and function. Adv Nutr, 2012. 3 .

[7] Dawodu, A. and R. C. Tsang, Maternal Vitamin D Status: Effect on Milk Vitamin D Content and Vitamin D Status of Breastfeeding Infants. Advances in Nutrition, 2012. 3 (3): p. 353-361.

[8] Jones, K. D., J. A. Berkley, and J. O. Warner, Perinatal nutrition and immunity to infection. Pediatric Allergy and Immunology, 2010. 21 (4p1): p. 564-576.

[9] Arimond, M., Simple food group diversity indicators predict micronutrient adequacy of women's diets in 5 diverse, resource-poor settings. J Nutr, 2010. 140.

[10] Saaka, M., Maternal dietary diversity and infant outcome of pregnant women in Northern Ghana. International Journal of Child Health and Nutrition, 2013. 1 (2): p. 148-156.

[11] Nguyen, P. H., et al., Maternal and Child Dietary Diversity Are Associated in Bangladesh, Vietnam, and Ethiopia, 2. The Journal of nutrition, 2013. 143 (7): p. 1176-1183.

[12] Berihun, S., G. M. Kassa, and M. Teshome, Factors associated with underweight among lactating women in Womberma woreda, Northwest Ethiopia; a cross-sectional study. BMC Nutrition, 2017.3 (1): p. 46.

[13] Biru, K., A. Jima, and S. Abeya, Prevalence of Chronic Energy Malnutrition and maternal health service utilizations among lactating mothers in Adama district, Oromia Region, Eastern Ethiopia. Journal of Food Processing and Technology, 2017. 8 (1).

[14] Haileslassie, K., A. Mulugeta, and M. Girma, Feeding practices, nutritional status and associated factors of lactating women in Samre Woreda, South Eastern Zone of Tigray, Ethiopia. Nutr J, 2013. 12: p. 28.

[15] Hundera, D., et al., Nutritional status and associated factors among lactating mothers in Nekemte Referral Hospital and Health Centers, Ethiopia. Int J Nutr Food Sci, 2015. 4 (2): p. 216-22.

[16] Sitotaw, I. K., K. Hailesslasie, and Y. Adama, Comparison of nutritional status and associated factors of lactating women between lowland and highland communities of District Raya, Alamata, Southern Tigray, Ethiopia. BMC Nutrition, 2017. 3 (1): p. 61 .

[17] FAO and USAID, Food and Nutrition Technical Assistance III Project (FANTA). 2016: Rome.
[18] Hoddinott, J. and Y. Yohannes, Dietary Diversity as a Food Security Indicator. 2002, Washington, D. C: Food and Nutrition Technical Assistance Project, Academy for Educational Development.

[19] Black, R. E., Maternal and child undernutrition and overweight in low-income and middle-income countries. Lancet, 2013. 382.

[20] UNICEF, Infant and Young Child feeding response. 2010.

[21] Muller O. and Krawinkel M., Malnutrition and health in developing countries. CMAJ, 2005. 173: p. 279-286.

[22] Alemayehu, M., A. Argaw, and G. Mariam A., Factors Associated with Malnutrition among Lactating Women in Subsistence Farming Households from Dedo and SeqaChekorsa Districts, Jimma Zone, 2014. iiste-developing country studies $2015.5(21)$.

[23] Nakamori, M., Nutritional status of lactating mothers and their breast milk concentration of iron, zinc, and copper in rural Vietnam. J Nutr Sci Vitaminol (Tokyo), 2009. 55.

[24] Haddis F., Levels, Differentials and Determinants of Malnutrition among Women in Ethiopia. Addis Ababa: School of Graduate Studies Addis Ababa University; 2008.

[25] Hindin MJ., Women's Autonomy, Status, and Nutrition in Zimbabwe, Zambia, and Malawi. Maryland: ORC Macro Calverton, 2005.

[26] Girma, W. and T. Genebo, Determinants of the nutritional status of mothers and children in Ethiopia. 2002, Calverton: ORC Macro.

[27] Khan, Y. M. and A. Khan, A study on factors influencing the nutritional status of lactating women in Jammu, Kashmir and Ladakh regions. Inter J Advancements Res Technol, 2012. 1.

[28] Henjum, S., Low dietary diversity and micronutrient adequacy among lactating women in a peri-urban area of Nepal. Public Health Nutr, 2015. 18.

[29] Mtumwa, A. and E. Paul, Determinants of undernutrition among women of reproductive age in Tanzania mainland. South African Journal of Clinical Nutrition, 2016. 29 (2): p. 75-78.

[30] Nguyen, P., et al., Maternal and Child Dietary Diversity Are Associated in Bangladesh, Vietnam, and Ethiopia. Community and International Nutrition, 2013. 143: p. 1176-1183.

[31] Bain, L. E., Malnutrition in sub-Saharan Africa: burden, causes, and prospects. Pan Afr Med J, 2013. 15. 